\title{
Correction to: Asymptotic Properties of Least Squares Estimators and Sequential Least Squares Estimators of a Chirp-like Signal Model Parameters
}

\author{
Rhythm Grover ${ }^{1} \mathbb{D} \cdot$ Debasis Kundu $^{2} \cdot$ Amit Mitra $^{2}$
}

Published online: 23 July 2021

○) Springer Science+Business Media, LLC, part of Springer Nature 2021

\section{Correction to: Circuits, Systems, and Signal Processing https://doi.org/10.1007/s00034-021-01724-7}

In this article, the captions to Figs. 16, 17 and 18 were inadvertently swapped. The corrected captions are: Fig. 16, "Speech Signal data sets: "EEE" and "UUU"; Observed data (red solid line) and fitted signal (blue dashed line). The sub-plots on the left represent chirp model fitting and those on the right represent chirp-like model fitting', Fig. 17, 'Simulated data' and Fig. 18, 'Simulated data signal along with estimated signal using chirp model'.

Also, Fig. 19 was missing from this article; the figure should have appeared as shown below.

The original article can be found online at https://doi.org/10.1007/s00034-021-01724-7.

Rhythm Grover

rhythm.grover91@gmail.com; rhythm@iitk.ac.in

Debasis Kundu

kundu@iitk.ac.in

Amit Mitra

amitra@iitk.ac.in

1 Theoretical Statistics and Mathematics Unit, Indian Statistical Institute, Delhi 110016, India

2 Department of Mathematics and Statistics, Indian Institute of Technology, Kanpur 208016, India 


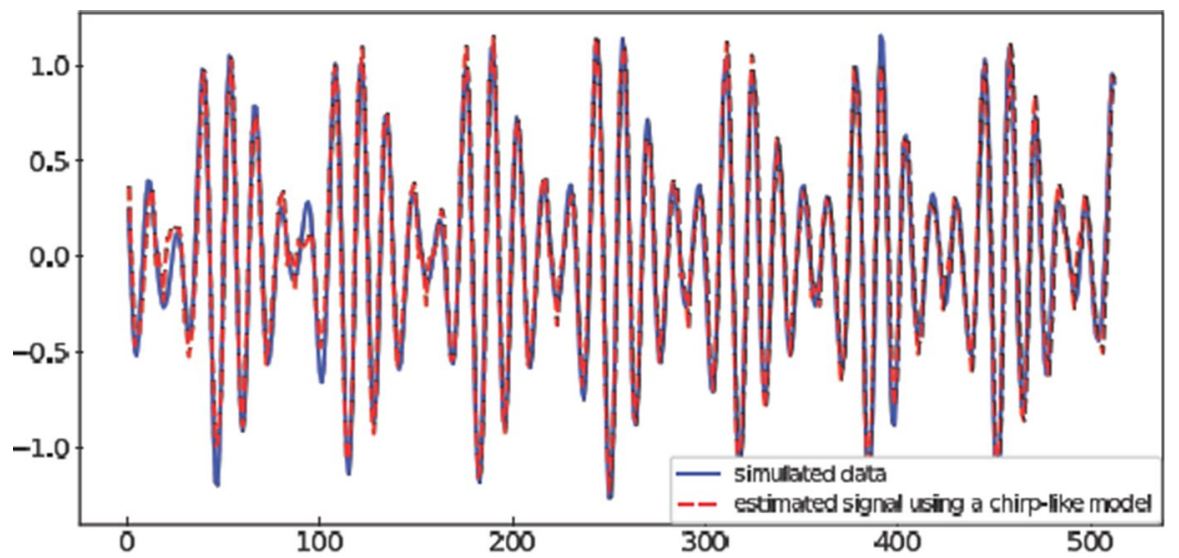

Fig. 19 Simulated data signal along with estimated signal using chirp-like model

Publisher's Note Springer Nature remains neutral with regard to jurisdictional claims in published maps and institutional affiliations. 